\title{
Resilience to Chronic Stress Is Mediated by Hippocampal Brain-Derived Neurotrophic Factor
}

\author{
Dekel Taliaz, Assaf Loya, Roman Gersner, Sharon Haramati, Alon Chen, and Abraham Zangen \\ Department of Neurobiology, Weizmann Institute of Science, Rehovot 76100, Israel
}

Chronic stress is a trigger for several psychiatric disorders, including depression; however, critical individual differences in resilience to both the behavioral and the neurochemical effects of stress have been reported. A prominent mechanism by which the brain reacts to acute and chronic stress is activation of the hypothalamic-pituitary-adrenal (HPA) axis, which is inhibited by the hippocampus via a polysynaptic circuit. Alterations in secretion of stress hormones and levels of brain-derived neurotrophic factor (BDNF) in the hippocampus were implicated in depression and the effects of antidepressant medications. However, the potential role of hippocampal BDNF in behavioral resilience to chronic stress and in the regulation of the HPA axis has not been evaluated. In the present study, Sprague Dawley rats were subjected to 4 weeks of chronic mild stress (CMS) to induce depressive-like behaviors after lentiviral vectors were used to induce localized BDNF overexpression or knockdown in the hippocampus. The behavioral outcome was measured during 3 weeks after the CMS procedure, then plasma samples were taken for measurements of corticosterone levels, and finally hippocampal tissue was taken for BDNF measurements. We found that hippocampal BDNF expression plays a critical role in resilience to chronic stress and that reduction of hippocampal BDNF expression in young, but not adult, rats induces prolonged elevations in corticosterone secretion. The present study describes a mechanism for individual differences in responses to chronic stress and implicates hippocampal BDNF in the development of neural circuits that control adequate stress adaptations.

\section{Introduction}

Stressful life events are associated with the onset of psychiatric disorders, including major depression (Kendler et al., 1999). Resilience to stress have been associated with the capacity to constrain stress-induced increases in corticotrophin-releasing hormone and corticosterone (CORT) [a hormone secreted by the adrenal gland and considered a marker for hypothalamic-pituitary-adrenal (HPA) activation] through an elaborated negative feedback system (Sapolsky et al., 1984; Charney, 2004). This negative feedback system involves optimal function and balance of glucocorticoid and mineralocorticoid receptors in the hippocampus (Sapolsky et al., 1991; Ridder et al., 2005; de Kloet et al., 2007). Importantly, critical individual differences in resilience to both the behavioral and the neurochemical effects of stress have been reported (Feder et al., 2009). Krishnan et al. (2007) found that brain-derived neurotrophic factor (BDNF) expression in the ventral tegmental area-nucleus accumbens (VTANAc) pathway plays a critical role in such resilience and that BDNF knockdown (KD) within the VTA ameliorates depressing effects of social defeat (Krishnan et al., 2007). In the hippocampus, however, the role of BDNF may be opposite, as we have

Received Oct. 28, 2010; revised Jan. 20, 2011; accepted Jan. 24, 2011.

This research was supported by the Israel Science Foundation Grant 1486/10 and by the Israel Ministry of Health Grant 3-5943. We thank Noga Zilkha, Yamit Beny, Aldema Shoshana Chen, Vini Nagaraj, Jee-eun Cho, and Noam Barnea for their contribution to the research.

The authors declare no competing financial interests.

Correspondence should be addressed to Dr. Abraham Zangen, Department of Neurobiology, Weizmann Institute of Science, Rehovot 76100, Israel. E-mail: a.zangen@weizmann.ac.il.

DOI:10.1523/JNEUROSCI.5725-10.2011

Copyright $\odot 2011$ the authors $\quad 0270-6474 / 11 / 314475-09 \$ 15.00 / 0$ found that BDNF KD within the dorsal dentate gyrus (dDG) per se induces depressive-like behaviors (Taliaz et al.,2010). Indeed, BDNF seems to exert very different effects in different brain regions, in response to stress. Although stress decreases BDNF expression in the hippocampus (Smith et al., 1995), it increases BDNF expression in the NAc (Nestler and Carlezon, 2006).

The role of hippocampal BDNF in resilience to stress can be assessed by comparing the effects of chronic mild stress (CMS) in adult and young rats. CMS in adult rats reduces BDNF expression in the dorsal hippocampus (Grønli et al., 2006; Toth et al., 2008; Gersner et al., 2010) and causes reductions in sucrose preference, sexual behavior, and brain stimulation reward, indicating a state of anhedonia (Muscat and Willner, 1992; Grønli et al., 2006; Toth et al., 2008). Conversely, young rats show resilience to the anhedonic effect of CMS, and, unlike adult rats, their hippocampal BDNF levels remain intact (Toth et al., 2008). This seems to contradict a common notion in humans, because childhood trauma is a potent risk factor for developing depression in adulthood (Heim et al., 2008). It is therefore necessary to further elucidate the interaction between chronic stress, hippocampal BDNF, and behavioral outcomes.

Childhood trauma is associated with altered dynamics of the HPA axis in adulthood (Heim et al., 2008). Indeed, plasma levels of CORT are altered in adult rats that were maternally separated or later exposed to CMS in early life (Francis et al., 2002; Lippmann et al., 2007; Toth et al., 2008). Obviously, the degree of stress plays an important role; rats that experienced long, but not short, maternal separation showed an increased elevation of CORT in response to acute stress in adulthood (Francis et al., 2002; Lippmann et al., 2007). Additionally, long (but not short) 
maternal separations resulted in significant decrease of BDNF in mature rats (Lippmann et al., 2007). These reports, together with our recent finding that localized BDNF KD within the dDG causes depressive-like behaviors, suggest that resilience of young rats to the behavioral effects of CMS is an outcome of their ability to maintain normal hippocampal BDNF expression. Here, we have examined this hypothesis using BDNF KD and tested whether overexpression (OE) of BDNF in adult rats can induce resilience to CMS. Finally, the potential role of hippocampal BDNF expression in HPA axis activity, as measured by spontaneous and stress-induced CORT levels, was evaluated in young and adult rats.

\section{Materials and Methods}

\section{Cloning lentiviral BDNF overexpression vectors}

For BDNF OE, BDNF was amplified by $\mathrm{PCR}$ using Bio-X-act DNA polymerase (Bioline) from the same pcDNA3.1 $(-)$ plasmid (Invitrogen) that we have used previously to express the rat BDNF coding sequence (Taliaz et al., 2010). The following primers were used: $5^{\prime}$-ATTTGGATCCTTCCACCAGGTGAGAAGAGTG-3' (forward) and 5' -TTTAGGATCCTATCTTCCCCTTTTAATG-3' (reverse). BamHI (Fermentas) restriction sites were introduced at the $5^{\prime}$ of each primer, which allowed for the insertion of the BDNF sequence downstream to the cytomegalovirus (CMV) promoter of the pCSC-SP-PW-IRES-GFP plasmid [lentiviral vector (LV) transfer plasmid] and upstream to the IRES-GFP sequence (kindly provided by Dr. Inder M. Verma, The Salk Institute for Biological Studies, La Jolla, CA).

\section{High titer lentiviral preparation}

LVs were prepared as we described previously (Taliaz et al., 2010), with slight modifications for the BDNF OE vector (LV-BDNF). HEK293T cells were cultured with DMEM (Invitrogen) containing 10\% fetal calf serum (FCS) (Invitrogen). Cells were then split into $15 \mathrm{~cm}$ plates, precoated with $20 \mu \mathrm{g} / \mathrm{ml}$ of poly-L-lysine (Sigma-Aldrich) to reach $60 \%$ confluency. On the transfection day, medium was replaced with DMEM (which did not include FCS) and cells were transfected using polyethyleneimine (PEI). To knockdown BDNF expression, we used our previously described LV (Taliaz et al., 2010), which expresses short hairpin (sh) RNA complementary to the rats BDNF coding sequence (LV-sh$\mathrm{BDNF}$ ). For LV-shBDNF (KD) preparation, a DNA mixture containing $26 \mu \mathrm{g}$ of transfer plasmid, $16.9 \mu \mathrm{g}$ of packaging plasmid [encoding viral Gag and Pol proteins (pCMV $\Delta$ R8.91)], $9.1 \mu \mathrm{g}$ of envelope plasmid [encoding the envelope of vesicular stomatitis virus (pCI)], and $78 \mu \mathrm{l}$ of 1 $\mathrm{mg} / \mathrm{ml}$ PEI in a total volume of $1.5 \mathrm{ml}$ of DMEM was prepared. For the LV-BDNF (OE) preparation, a DNA mixture containing $12 \mu \mathrm{g}$ of transfer plasmid, $7.8 \mu \mathrm{g}$ of packaging plasmid [encoding viral Gag and Pol proteins (pMDL)], $3 \mu \mathrm{g}$ of plasmid expressing Rev (pRSV-Rev), $4.2 \mu \mathrm{g}$ of envelope plasmid [encoding the envelope of vesicular stomatitis virus (pCI)], and $40.5 \mu \mathrm{l}$ of $1 \mathrm{mg} / \mathrm{ml}$ PEI in a total volume of $1.5 \mathrm{ml}$ of DMEM was prepared. The mixtures were incubated for $10 \mathrm{~min}$ at room temperature and then added dropwise to plates containing HEK293T cells. Sixteen hours after transfection, the medium was replaced with DMEM supplemented with 10\% FCS. Forty-eight and $72 \mathrm{~h}$ after transfection, medium was collected, cleared by low-speed centrifugation, filtered through $0.45 \mu \mathrm{m}$-pore-size filter, and ultracentrifuged at 25,000 rpm for $2 \mathrm{~h}$ in $4^{\circ} \mathrm{C}$ (Beckman Coulter) to obtain high titer viral stocks. The pellet was resuspended in $0.1 \mathrm{ml}$ of PBS, and aliquots were stored at $-80^{\circ} \mathrm{C}$ until further use. All LVs are expressing green fluorescent protein (GFP) as a marker. To determine titer of the viral stocks, $10^{5}$ HEK293T cells were infected with 5 or $20 \mu \mathrm{l}$ of viral stock. After $48 \mathrm{~h}$, cells were fixed and then analyzed with fluorescence activated cell sorting, and GFP-positive cells were detected. Our LV stock titers are expressed as transducing units (TU) per milliliter and ranged in the order of $10^{9} \mathrm{TU} / \mathrm{ml}$.

\section{Validation of $L V-B D N F$ activity in vitro}

HEK293T cell lines were grown to $60 \%$ confluency and infected with LV-BDNF or LV-GFP ( $\mathrm{n}=3$ per treatment). Cells were allowed to grow to $100 \%$ confluency, and medium was replaced. Twenty-four hours later, medium was collected and stored at $-20^{\circ} \mathrm{C}$ for further analysis, and cells were harvested, rinsed twice with ice-cold PBS, and underwent lysis with radioimmunoprecipitation assay buffer $(140 \mathrm{~mm} \mathrm{NaCl}, 20 \mathrm{~mm}$ Tris, $\mathrm{pH}$ $7.4,10 \%$ glycerol, $1 \%$ Triton X-100, 0.5\% deoxycholate, $0.1 \%$ SDS, 2 mM EDTA, $1 \mathrm{~mm}$ PMSF, and leupeptin at $20 \mu \mathrm{g} / \mathrm{ml}$ ). Lysates were centrifuged at $14,000 \mathrm{rpm}$ for $10 \mathrm{~min}$ at $4^{\circ} \mathrm{C}$, the pellet was discarded, and aliquots of the supernatant were stored at $-20^{\circ} \mathrm{C}$ for further analysis. BDNF protein levels were determined using DuoSet ELISA development system ( R \& D Systems) (Taliaz et al., 2010). Results were normalized per cell number for medium samples or per total protein concentration (Bradford assay; Bio-Rad) for protein extraction samples.

\section{Validation of $L V-B D N F$ activity in vivo}

To verify the efficiency of BDNF OE in vivo, rats were divided into three different groups; two groups were microinjected with either LV-GFP or LV-BDNF to the $\mathrm{dDG}$ as described below, and one group was not treated ( $n=5-7$ per group). After 2 weeks, rats were decapitated and hippocampal samples were taken for BDNF measurements using sandwich ELISA as we described previously (Toth et al., 2008; Taliaz et al., 2010).

\section{Validation of $L V-$ shBDNF}

BDNF knockdown was validated in vitro and in vivo similarly to the LV-BDNF validation and as we described recently (Taliaz et al., 2010).

\section{Animals}

Ten-week-old (adult) and 21-d-old (young) male Sprague Dawley rats (Harlan Laboratories) were housed individually on a standard $12 \mathrm{~h}$ light/ dark schedule (lights on at 7:00 A.M). Food and water were available ad libitum, except for the CMS experiment, which included food and water deprivation manipulations as detailed below. All animals were handled according to the regulations formulated by the Institutional Animal Care and Use Committee, which are in complete accordance with National Institutes of Health Guidelines for Care and Use of Laboratory Animals.

\section{Intracranial surgery}

Rats were anesthetized with ketamine $(170 \mathrm{mg} / \mathrm{kg})$ and acepromazine $(1.7 \mathrm{mg} / \mathrm{kg})$ and placed in a stereotaxic frame. Stainless steel guide cannulae (Plastics One) were implanted bilaterally into the dDG of the hippocampus of adult rats, using the following coordinates (relative to bregma and according to the atlas of Paxinos and Watson, 1998) as we described previously (Taliaz et al., 2010): $-3.8 \mathrm{~mm}$ anteroposterior, $+2.4 \mathrm{~mm}$ mediolateral, and $-1.95 \mathrm{~mm}$ dorsoventral ( $1.5 \mathrm{~mm}$ above the infection site) at a lateral angle of $10^{\circ}$. The young rats were not implanted with guide cannulae because of the length of the study and their expected growth. Therefore, microinjection for BDNF knockdown (or control LV) in young rats were performed only once during the surgery. The coordinates for the young rats were assessed and optimized by preliminary trials and histological evaluations and were as follows (relative to bregma): $-3.2 \mathrm{~mm}$ anteroposterior, $+1.65 \mathrm{~mm}$ mediolateral, and -3.4 mm dorsoventral.

\section{Microinjection of $L V-s h B D N F$ or $L V-B D N F$}

In the groups of young rats, bilateral microinjections ( $2 \mu$ l over 4 min) of either LV-shBDNF (to induce knockdown) or LV-shSCR (scrambled control) were performed during the intracranial surgery into the coordinates found in the validation process (see above). Injectors were left intact for $2 \mathrm{~min}$ after completing the injection to ensure adequate diffusion from the injector tip. Four days after the infection, rats were subjected to the CMS procedure or left at their home cage. Behavioral analysis was initiated $1 \mathrm{~d}$ after completion of the CMS procedure.

In the groups of adult rats, bilateral microinjections ( $2 \mu \mathrm{l}$ over $4 \mathrm{~min})$ of either LV-shBDNF, LV-shSCR, LV-BDNF, or LV-GFP were performed into the aforementioned coordinates using a dual-channel MAB 40 microdialysis pump (Microbiotech/SE AB). Microinjections were repeated three times every other day as described previously (Taliaz et al., 2010).

\section{Chronic mild stress}

Young and adult rats were subjected to a slightly more intensive CMS procedure than the one we described previously (Toth et al., 2008). Rats were subjected to several different and unpredictable mild stressors during a 4 week period. The procedure included the following: a $45^{\circ}$ cage tilt 


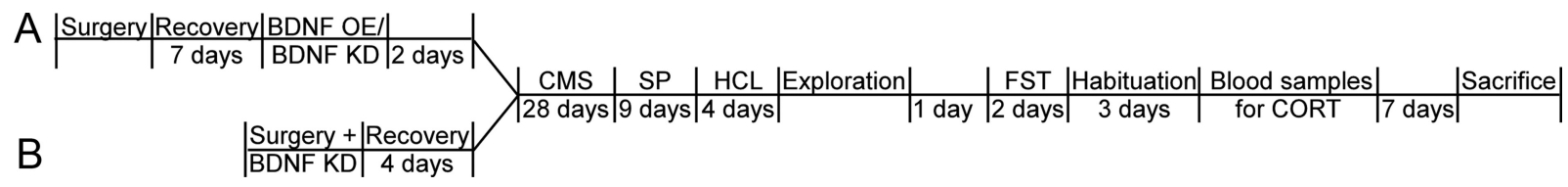

Figure 1. Timeline of experimental procedures. BDNF expression was altered at the $\mathrm{dDG}$ of adult $(\boldsymbol{A})$ and young $(\boldsymbol{B})$ rats (OE and KD). Then, rats were subjected to 4 weeks of $C \mathrm{CMS}$ or left undisturbed. Then, behavioral measures were initiated and included sucrose preference (SP), home-cage locomotion (HCI), exploration of a novel environment, and an FST. After the behavioral measurements and $3 \mathrm{~d}$ of habituation, blood samples were drawn twice for CORT measurements: before and immediately after acute stress. Seven days later, rats were killed, and brains were removed for BDNF analysis.

twice a week for 5 and $21 \mathrm{~h}$, low-intensity stroboscopic light (flicker) twice a week for 7 and $17 \mathrm{~h}$, intermittent white noise $(80 \mathrm{~dB})$ twice a week for 5 and $14 \mathrm{~h}$, soiled cages (by adding $100 \mathrm{ml}$ of water to the home cage) for $21 \mathrm{~h}$ per week, food and water deprivation three times a week for 21 and $24 \mathrm{~h}$, paired housing for $7 \mathrm{~h} /$ week, and lights on overnight for 2 nights each week. The water deprivation for $21 \mathrm{~h}$ was followed by exposure to empty water bottle for $1 \mathrm{~h}$; the food deprivation for $24 \mathrm{~h}$ was followed by $2 \mathrm{~h}$ in which $0.5 \mathrm{~g}$ of food pellet was provided. This schedule of stressors was applied for a 1 week period and repeated over 4 weeks.

\section{Sucrose preference test}

Sucrose preference test was performed as described previously (Toth et al., 2008; Gersner et al., 2010; Taliaz et al., 2010). Rats had access to two drinking spouts positioned side-by-side at the rear of the cage. Fluid consumption was recorded by weighing the bottles every morning between 9:30 A.M. and 10:00 A.M. Sucrose solution (0.2\% sucrose in tap water) was placed in one bottle and tap water in the other. Rats were tested for $4 \mathrm{~d}$, followed by $1 \mathrm{~d}$ of tap water only, and then bottle positions were switched and rats were tested for an additional $4 \mathrm{~d}$ to control for side preference. Sucrose preference for each rat was defined as the average ratio between sucrose to water consumption for the whole period $(8 \mathrm{~d})$ excluding the first measure at each side.

\section{Home-cage locomotion}

Home-cage locomotion was assessed as described previously (Toth et al., 2008; Gersner et al., 2010; Taliaz et al., 2010) by an automated chronic monitoring of locomotion in the home cages using a computerized Inframot system (TSE), which is based on infrared sensors located above the cage of each rat. Mobility during the dark period (over $12 \mathrm{~h} /$ night) was analyzed over $4 \mathrm{~d}$.

\section{Exploration and novelty-induced behavior}

Exploration test was performed in a novel environment as described previously (Toth et al., 2008; Gersner et al., 2010; Taliaz et al., 2010). Rats were placed in a $40 \times 40 \mathrm{~cm}$ exploration box (ActiMot System Activity Chamber; TSE), and the distance traveled was recorded automatically over $10 \mathrm{~min}$. The distance traveled was estimated by beam breaks of 32 photo beam pairs, separated by $2.5 \mathrm{~cm}$ and placed $3 \mathrm{~cm}$ above the floor. The exploration box was thoroughly cleaned between tests.

\section{Forced swim test}

A modified forced swim test (FST) was conducted in a cylindrical tank (40 $\mathrm{cm}$ high and $18 \mathrm{~cm}$ in diameter; constructed at the Weizmann Institute), as we described previously (Toth et al., 2008; Gersner et al., 2010; Taliaz et al., 2010). The water temperature was kept at $25^{\circ} \mathrm{C}\left(2^{\circ} \mathrm{C}\right.$ above room temperature), and the water level was such that the rat could not touch the bottom with its hindpaws. Rats were exposed to the swim tank for $10 \mathrm{~min}$ at the first day and $5 \mathrm{~min}$ at the second day and were videotaped. Video films of the second day of each FST session were analyzed by a software developed in our laboratory, which continuously examines movement of pixels adjacent to the rat limbs and detects fine alterations in mobility throughout the test (Gersner et al., 2005, 2009). This method yields a sensitive analysis of more information than the standard scoring protocols.

\section{Corticosterone measurements}

All rats were handled and habituated for 3 d, between 9:00 A.M. and 11:00 A.M., when CORT rates are at a basal level (Dhabhar et al., 1993). This procedure included carrying each rat home cage from its room to the room where the blood draw was to take place and handling the rats with minimal stress. On day 4, blood was drawn from each rat twice: first for baseline and $1 \mathrm{~h}$ later for stress measurements. For baseline measurements, each rat was taken individually, whereas in the stress condition, the rats were taken in groups of five and each rat was left on an elevated platform $(12 \times 12 \mathrm{~cm}, 130 \mathrm{~cm}$ height $)(X u$ et al., 1998) before blood was drawn. In both conditions, rats were anesthetized using $\mathrm{CO}_{2}$ (Fomby et al., 2004; Altholtz et al., 2006), and, for baseline measurement, the procedure took no longer than 3 min overall for each rat individually; thus, CORT levels should not be elevated (Dhabhar et al., 1993). One milliliter samples of blood were drawn from the rat's retro-orbital plexus (Fomby et al., 2004) and centrifuged $(1500 \times g, 15 \mathrm{~min})$, and $0.3 \mathrm{ml}$ plasma samples were stored in $-80^{\circ} \mathrm{C}$. CORT levels were measured by ELISA using corticosterone EIA kit (Cayman Chemical).

\section{In vivo $B D N F$ measurements}

$B D N F$ ELISA. To quantify BDNF protein levels by ELISA, rats were decapitated, and their brains were extracted, immediately frozen in isopropanol, and stored at $-80^{\circ} \mathrm{C}$. Bilateral tissue punches were obtained from coronal sections generated by a manual cut within the cryostat environment (at $-20^{\circ} \mathrm{C}$; Leica CM $3050 \mathrm{~S}$ ) and according to a rat brain atlas (Paxinos and Watson, 1998). The coronal section used for punches of the $\mathrm{dDG}$ was taken from -2.3 to $-4.3 \mathrm{~mm}$ from bregma, as we described previously (Toth et al., 2008; Taliaz et al., 2010). Protein extraction and sandwich ELISA were performed, and BDNF concentration was normalized per total protein or per tissue weight (Toth et al., 2008; Taliaz et al., 2010). Because similar results (comparing groups) were obtained when normalized in both ways, the results are presented after normalization to total protein levels.

BDNF immunostaining. Rats were deeply anesthetized and transcardially perfused using PBS supplemented with $4 \mathrm{U} / \mathrm{ml}$ heparin (Merck Biosciences), followed by fresh $2.5 \%$ paraformaldehyde (PFA) (J. T. Baker) supplemented with 5\% sucrose (J. T. Baker) in PBS. Brains were removed, postfixed overnight, and allowed to sink in 2.5\% PFA supplemented with $30 \%$ sucrose in PBS solution. Coronal sections $(30 \mu \mathrm{m})$ were collected using a cryostat and placed into 24 -well plates containing $0.01 \%$ azide (Sigma-Aldrich) in PBS and were stored at $4^{\circ} \mathrm{C}$ until further use. Next, flouting sections were incubated for $1 \mathrm{~h}$ with $20 \%$ normal horse serum (NHS) (Vector Laboratories) and 0.05\% saponin (SigmaAldrich). Sections were then covered with anti-BDNF antibodies (Alomone Labs) diluted 1:300 in PBS containing 0.05\% saponin and 2\% NHS at room temperature. The following day, slices were washed three times with PBS and incubated for $1 \mathrm{~h}$ with 1:200 cyanine-3-conjugated donkey anti-rabbit secondary antibodies (Jackson ImmunoResearch). Sections were washed and were then covered with Hoechst solution (1:2000; Sigma-Aldrich) for 30-60 s to mark the nuclei of the cells. As a control for nonspecific staining, sections were incubated in identical conditions, without the corresponding primary antibody. Photomicrographs were taken using Nikon E800 microscope. BDNF, GFP, and Hoechst immunoreactivity were quantified using Image Pro Plus 4.5 software (Media Cybernetics) by measuring the intensity per unit surface area at the dDG. Four hippocampal sections per rat were used for this analysis.

\section{Experimental procedures}

After validation of LV efficiency in vitro and in vivo (see Results), all procedures and behavioral assessments were performed in all groups (young and adult) in the same order as described in the timeline (Fig. 1). 
Experiment 1: effect of hippocampal BDNF overexpression on behavioral outcomes of CMS in adult rats. We used four groups of rats $(n=8-13$ per group) to evaluate behavioral effects of hippocampal BDNF OE and CMS in adult rats. A control vector or a vector that expressed the rat BDNF coding sequence was injected bilaterally into the $\mathrm{dDG}$ of adult rats. Then, half of the rats of each group were exposed to the CMS procedure, whereas the other half remained undisturbed at their home cage for the same period $(2 \times 2$ design; statistical factors are CMS and BDNF OE). The battery of behavioral measures described above was then used to identify depressive-like symptoms in each group. After the behavioral measures, in each experimental condition, part of the rats was used for quantitative BDNF ELISA measures (six to seven per group), and another part was used for BDNF immunohistochemistry (two to four per group).

Experiment 2: effect of hippocampal BDNF knockdown on the resilience of young rats to CMS. The LV-shBDNF or control vectors were microinjected into the dDG of young rats to induce BDNF KD (or merely GFP expression), as described above. Then, half of the rats within each group were subjected to the CMS procedure, whereas the other half remained undisturbed at their home cage for the same period $(2 \times 2$ design; statistical factors are CMS and BDNF KD). The standard CMS procedure in young rats does not induce a significant reduction in sucrose preference (Toth et al., 2008), but the trends in the behavioral measures and the individual differences indicated that some of the young rats were not resilient to the CMS procedure. We have therefore divided the rats subjected to CMS and infected with the control LV into resilient and nonresilient based on their sucrose preference. Rats showing preference of sucrose/water ratio above 1.5 despite the CMS procedure were defined resilient. This experiment therefore included five different groups, because only the CMS-control LV group could be divided into resilient and nonresilient. Consequently, the $2 \times 2$ design for statistical analysis (as performed in experiment 1 ) was not feasible and comparison was made between all five experimental groups using one-way ANOVA $(n=8-22$ per group for each of the behavioral experiments, except for the home cage locomotion in which $n=8-15$ per group). After the behavioral measures, in each experimental condition, part of the rats was used for quantitative BDNF ELISA measures (six to eight per group), and another part was used for BDNF immunohistochemistry (two to five per group).

Experiment 3: effects of CMS and manipulations of hippocampal BDNF expression on corticosterone plasma levels of young and adult rats. To test whether manipulations of hippocampal BDNF expression in young or adult rats induce long-lasting effects on hippocampal regulation of the HPA axis, we have measured baseline and stress-induced alterations in CORT blood levels in groups of rats that were previously subjected to BDNF alterations (KD or OE) and/or CMS in young or adult ages.

Experiment $3 a$. In this experiment, we tested the long-lasting effect of hippocampal BDNF KD induced in young or adult rats on CORT plasma levels. We induced BDNF KD in the dDG of young and adult rats by microinjection of the above described LV-shBDNF. Therefore, this experiment included four groups of rats $(2 \times 2$ design; statistical factors are AGE and BDNF KD; $n=7-12$ per group). Eight weeks after the BDNF $\mathrm{KD}$, we have taken blood samples for CORT measurements before and after acute stress, as described above.

Experiment $3 b$. In this experiment, we tested the long-lasting effect of CMS on CORT plasma levels in young rats that were or were not resilient to CMS and that were or were not subjected to hippocampal BDNF KD. Eight weeks after BDNF KD, we have taken blood samples for CORT measurements before and after acute stress from the five groups of rats described in experiment 2 ( $n=7-12$ per group).

Experiment $3 c$. In this experiment, we tested the effect of BDNF OE and CMS on the CORT plasma levels of adult rats. Eight weeks after BDNF $\mathrm{OE}$, we have taken blood samples for CORT measurements before and after acute stress from the four groups of rats described in experiment 1 ( $n=7-12$ per group).

\section{Statistical analysis}

Results are expressed as mean \pm SEM. Analyses of behavioral experiments were first performed using two-way ANOVA $(\mathrm{LV} \times \mathrm{CMS})$, followed by post hoc Student's $t$ tests. Once the "resilience" criterion was
A

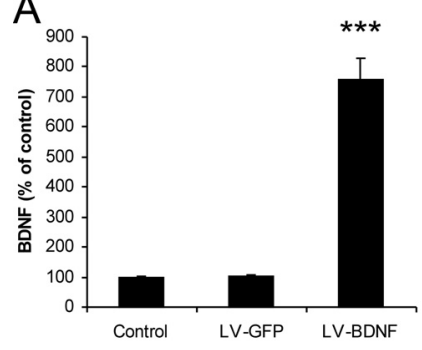

$\mathrm{B}$

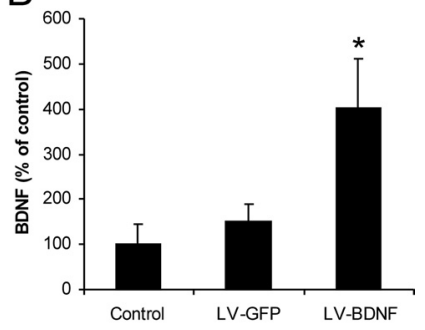

C

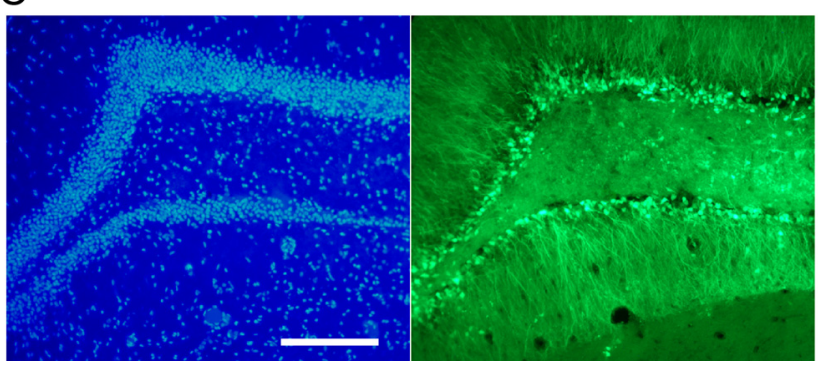

Figure 2. Validation of $L V$ infection and of BDNF overexpression. $A$, BDNF protein expression was measured in vitro in the medium of HEK293T cell line. Cells were infected with either LV-expressing GFP alone (LV-GFP) or LV that additionally expressed the coding axon of the rat BDNF gene (LV-BDNF). Secreted BDNF levels were measured in an HEK293T cell medium $48 \mathrm{~h}$ after infection and normalized per $10^{6}$ cell number. Data are presented as percentages of BDNF secreted from non-infected control cells. $\boldsymbol{B}$, In vivo measurements of BDNF expression in the dorsal hippocampus of adult rats microinjected with LV-GFP or with LV-BDNF into their dDG or in control non-injected adult rats (no infection). Levels of BDNF were measured using sandwich ELISA and are presented as percentages of BDNF extracted from the non-infected control group. Values are mean \pm SEM $\left({ }^{*} p<0.05,{ }^{* * *} p<0.001\right)$. C, Representative micrographs of the dDG of young rats injected with LV-GFP are presented. The selection of coordinates for microinjections was based on preliminary surgeries in young rats and inspection of the infected area under the microscope. The infected region and spread were detected using GFP (right). Cell nuclei were visualized using Hoechst (left). Scale bar, $200 \mu \mathrm{m}$. Validation of BDNF KD has been detailed by Taliaz et al. (2010), and BDNF alterations are presented in Figures $3 E$ and $4 E$.

established (in young rats receiving CMS and the control LV only), oneway ANOVA followed by Fisher's post hoc tests were used to compare behavioral differences between all groups. Similarly, CORT and BDNF levels were analyzed using two-way ANOVA $(\mathrm{LV} \times \mathrm{CMS}$ or $\mathrm{LV} \times \mathrm{AGE})$ followed by post hoc Student's $t$ tests, and, once the resilience criterion was established, one-way ANOVA followed by Fisher's post hoc tests were applied.

\section{GenBank accession number}

The BDNF GenBank accession number is NM_012513.

\section{Results}

\section{Validations of hippocampal BDNF alterations}

To reduce BDNF expression in the dDG (BDNF KD), we used our previously described LV-shBDNF (Taliaz et al., 2010). For BDNF $\mathrm{OE}$, we cloned the rat BDNF gene into LV and validated the efficiency of these LV-BDNF by infecting HEK293T cell line, which endogenously express very low levels of BDNF. BDNF levels in the medium of cells infected with the LV-BDNF were significantly higher than that of non-infected cells or of cells infected with the control LV-GFP (Fig. 2A). One-way ANOVA revealed a significant effect of BDNF OE $\left(F_{(2,6)}=91.94, p<0.001\right)$, and Fisher's post hoc analysis revealed that BDNF was significantly increased by infection with LV-BDNF but not with LV-GFP $(p<0.001)$. For in vivo validation, we microinjected the LVBDNF into the dDG of adult rats and measured local BDNF levels (Fig. $2 \mathrm{~B}$ ). One-way ANOVA revealed a significant effect of BDNF OE $\left(F_{(2,16)}=4.49, p<0.05\right)$, and Fisher's post hoc 
A
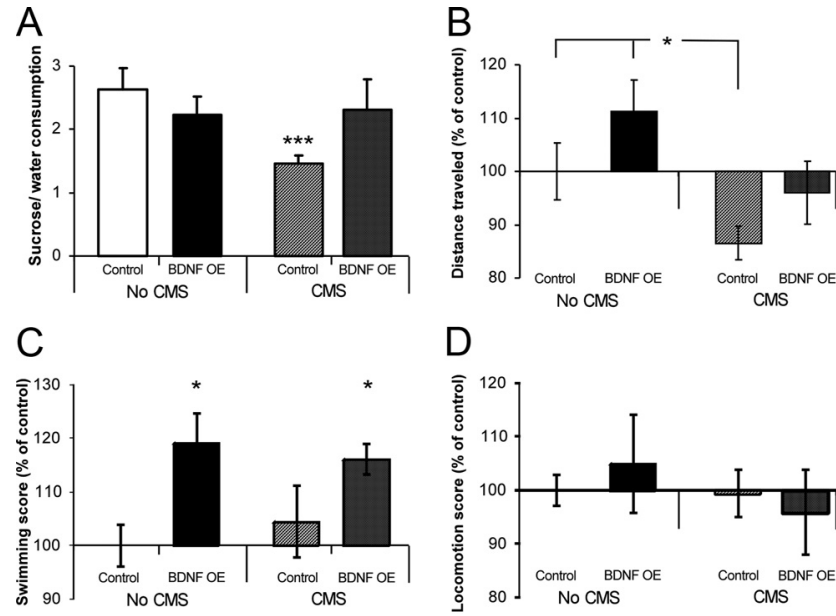

D
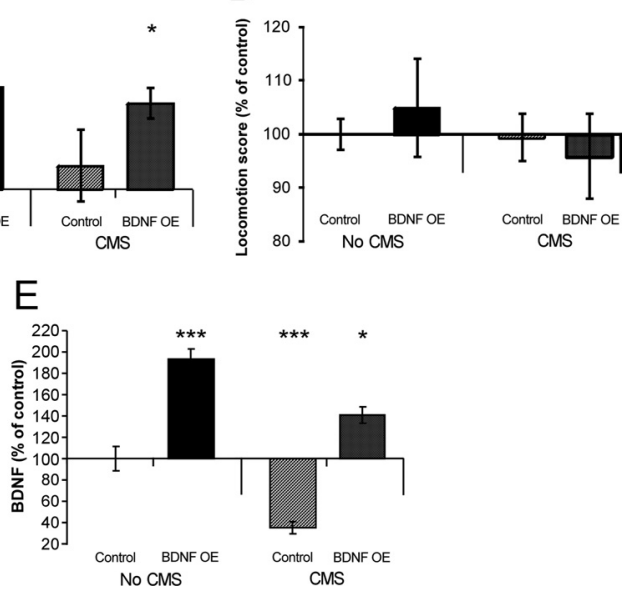

Figure 3. Behavioral effects of CMS and altered hippocampal BDNF expression in adult rats. $\mathrm{LV}-\mathrm{BDNF}(\mathrm{OE})$ or $\mathrm{LV}-\mathrm{GFP}$ (control) was injected into the $\mathrm{dDG}$ of adult rats, and their behavioral response to 4 weeks of CMS was evaluated according to the timeline detailed in Figure 1. Sucrose preference was assessed over a $9 \mathrm{~d}$ period using a $0.2 \%$ sucrose solution $(\boldsymbol{A})$. Distance traveled by the rats was automatically quantified in a 10 min exploration test that was conducted in a novel environment $(\boldsymbol{B})$, and home-cage locomotion was automatically assessed over $4 d(D)$. Finally, the activity of the rats in the FST was scored and analyzed automatically $(\boldsymbol{C})$. After the behavioral analysis, BDNF protein expression was measured in the dDG injected with LV-GFP or LV-BDNF $(\boldsymbol{E})$. Values are mean \pm SEM $\left({ }^{*} p<0.05,{ }^{* *} p<0.001\right.$, relative to the control group, which was infected with the control LV and not subjected to CMS).

analysis showed that BDNF levels were significantly higher in the group infected with LV-BDNF ( $p<0.05$ ) (Fig. $2 B$ ).

Because the young rats' brain continues development and growth and because standard rat brain atlases do not match the size of a 21-d-old rat, we first evaluated optimal coordinates for the dDG LV microinjection in 21-d-old rats using a dye and inspected the brain slices under the microscope (data not shown). Validation of infection and measurements of spread were performed using microinjections of LV-GFP into the determined coordinates and detection of GFP expression within the $\mathrm{dDG}$ in brain slices taken from these rats after 4 weeks (Fig. 2C).

Experiment 1: effect of hippocampal BDNF overexpression on behavioral outcomes of CMS in adult rats

We found that BDNF OE in the dDG of adult rats blocks depressive-like effects of CMS. Although CMS in adult rats caused a significant reduction in sucrose preference, the induction of BDNF OE in the $\mathrm{dDG}$ before the CMS procedure prevented such reduction in sucrose preference (Fig. $3 A$ ). A main effect of CMS $\left(F_{(1,33)}=4.52, p<0.05\right)$, and an interaction between CMS and LV infection $\left(F_{(1,33)}=5.59, p<0.05\right)$ were observed. Post hoc Student's $t$ tests comparing the different groups to the no-CMS control LV group revealed that CMS significantly reduced the sucrose preference of adult rats infected with the control LV-GFP $(p<0.001)$ but not of those infected with the LV-BDNF (Fig. 3A). In addition, whereas CMS in adult rats reduced the distance traveled in a novel environment, $\mathrm{BDNF}$ $\mathrm{OE}$ in the $\mathrm{dDG}$ before the CMS procedure blocked this reduction (Fig. $3 B)$. A main effect of CMS was observed in this test $\left(F_{(1,36)}=\right.$ $8.16, p<0.01)$, but post hoc Student's $t$ test revealed that CMS significantly reduced the distance traveled only by rats injected with control LV-GFP $(p<0.05)$ but not with LV-BDNF (Fig. $3 B)$. Finally, BDNF OE significantly increased the FST mobility score in both CMS and no-CMS groups (Fig. 3C). A main effect of BDNF OE was observed $\left(F_{(1,43)}=10.38, p<0.01\right)$, and post hoc Student's $t$ test comparing the different groups with the noCMS control LV group revealed that LV-BDNF increased the activity of rats in the FST (relative to the no-CMS control LV group) regardless of whether they were or were not subjected to CMS ( $p<0.05)$ (Fig. 3C). No significant differences between the various groups were observed in home-cage locomotion (Fig. $3 D$ ). Analysis of BDNF protein levels in the various groups of rats showed that CMS decreased and that BDNF OE effectively increased BDNF levels in the dDG (Fig. 3E). Main effects of CMS $\left(F_{(1,22)}=31.97, p<0.001\right)$ and of LV infection $\left(F_{(1,22)}=91.77\right.$, $p<0.01)$ were observed. Post hoc Student's $t$ tests comparing the different groups with the no-CMS control LV group revealed that CMS significantly decreased BDNF expression in the dDG of rats infected with control LV-GFP $(p<0.001)$. However, a significant increase of BDNF was observed in the dDG of rats subjected to CMS and infected with LV-BDNF ( $p<0.05)$. Finally, BDNF expression was significantly increased in the dDG of rats that were not subjected to CMS and were infected with LV-BDNF $(p<0.001)$ (Fig. 3E).

\section{Experiment 2: effect of hippocampal BDNF knockdown on the resilience of young rats to CMS}

The standard CMS procedure in young rats does not induce anhedonic effects such as reduction in sucrose preference (Toth et al., 2008) (see Fig. 6A). Indeed, in young rats, although a main effect of BDNF KD on sucrose preference was observed $(2 \times 2$ ANOVA, $\left.F_{(1,52)}=22.537, p<0.001\right)$, there was no significant effect of the CMS. However, the more intensive CMS procedure (including surgeries) used in the present study tended to affect the behavioral measures, and the individual variability indicated that some of the young rats were not resilient to this CMS procedure. Therefore, rats subjected to CMS and infected with the control LV were divided into resilient and nonresilient based on their sucrose preference. Rats showing sucrose/water ratio of above 1.5 (73.33\% of the rats) were defined resilient, and those showing lower sucrose preference $(26.66 \%$ of the rats) were defined nonresilient (Fig. 4A). One-way ANOVA showed a significant effect of treatment on sucrose preference $\left(F_{(4,63)}=4.75, p<\right.$ 0.01 ), and Fisher's post hoc analysis confirmed that the nonresilient CMS group showed a significant reduction in sucrose preference relative to the no-CMS control LV group $(p<0.05)$ (Fig. $4 A)$. In addition, home-cage locomotion and the distance traveled in a novel environment (exploration) of the resilient subgroup were not significantly different from those in the no-CMS control LV group (Fig. 4 B,D). Conversely, these behaviors (as well as sucrose preference) were significantly impaired in the nonresilient subgroup when compared with the no-CMS control LV group (Fig. $4 B, D)$. A significant effect of treatment was observed in the exploration of a novel environment test $\left(F_{(4,63)}=2.64, p<0.05\right)$. Fisher's post hoc analysis revealed that CMS induced a significant reduction in the distance traveled by nonresilient young rats when compared with the no-CMS control LV group ( $\mathrm{p}<0.01)$ (Fig. $4 B$ ). In addition, a significant effect of treatment was observed in home-cage locomotion scores $\left(F_{(4,46)}=7.17, p<0.01\right)$. Fisher's 

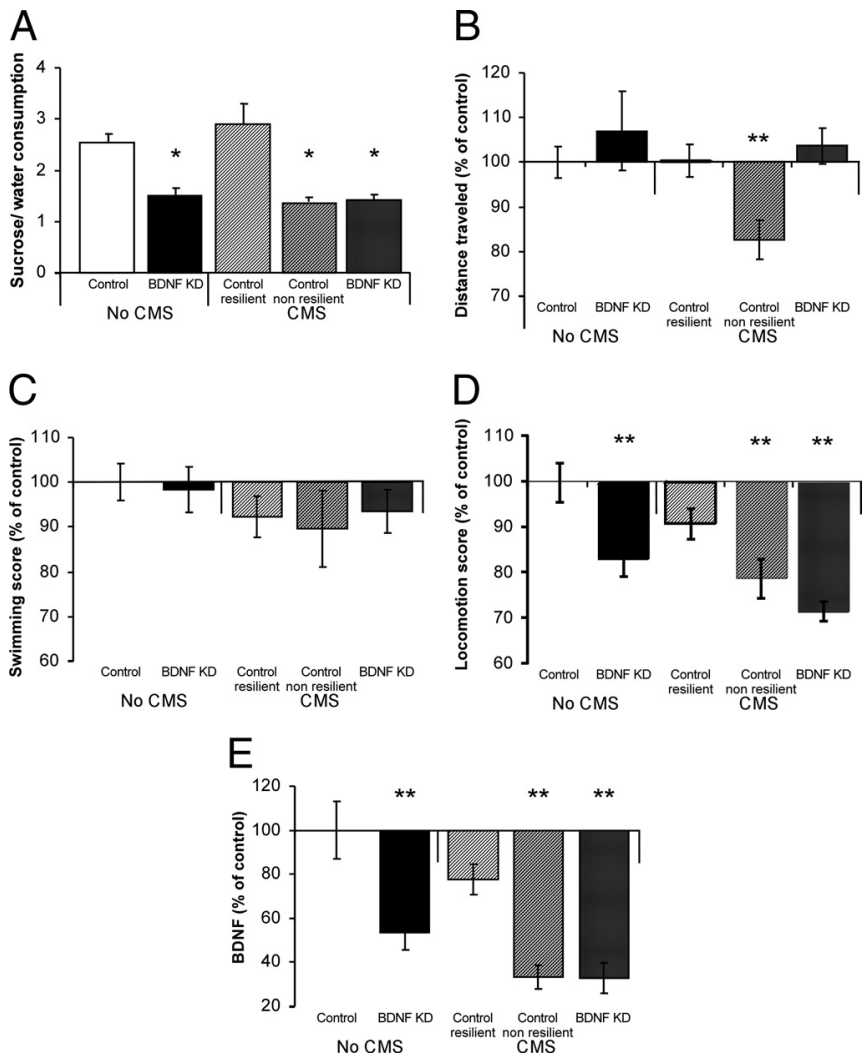

Figure 4. Behavioral effects of CMS and altered hippocampal BDNF expression in young rats. LV-shBDNF (KD) or LV-shSCR (control) was injected into the dDG of young rats, and their behavioral response to 4 weeks of CMS was evaluated (as detailed in Fig. 1). The young rats were further divided to CMS resilient and nonresilient groups (see Materials and Methods). The behavioral measurements include sucrose preference $(\boldsymbol{A})$, distance traveled at a novel environment $(\boldsymbol{B})$, activity at the forced swim test $(\boldsymbol{C})$, and home-cage locomotion (D). In addition, BDNF protein expression was measured in the $\mathrm{dDG}$ of young rats injected with LV-GFP or LV-BDNF $(\boldsymbol{E})$. Values are mean \pm SEM $\left({ }^{*} p<0.05\right.$, ${ }^{* *} p<$ 0.01 , relative to the control group, which was infected with the control LV and not subjected to (MS).

post hoc analysis revealed that the nonresilient CMS group showed a significant reduction in home-cage locomotion relative to the no-CMS control LV group ( $p<0.01$ ) (Fig. $4 D$ ).

Analysis of BDNF protein levels showed that CMS induced a reduction in $\mathrm{dDG}$ BDNF expression only in the behaviorally nonresilient subgroup (Fig. $4 E$ ), further supporting the role of dDG BDNF expression in resilience to chronic stress, as suggested by the BDNF OE experiment in adult rats (experiment 1 ). BDNF expression in the $\mathrm{dDG}$ of young rats was significantly affected by treatment $\left(F_{(4,27)}=\right.$ 7.95, $p<0.001$ ), and Fisher's post hoc analysis revealed that CMS induced a significant reduction in BDNF levels in the nonresilient (but not the resilient) young group when compared with the noCMS control LV group $(p<0.001)$ (Fig. $4 E)$.

In addition to the main effects described in this experiment, Fisher's post hoc analysis comparing the BDNF KD groups with the no-CMS control LV group revealed that BNDF KD within the dDG of the young rats significantly reduced local BDNF levels in both CMS $(p<0.001)$ and noCMS $(p<0.01)$ groups (Fig. $4 E$ ). In addition, BDNF KD caused a significant decrease in sucrose preference $(p<0.05)$ and home-cage locomotion $(p<0.01)$. These effects of dDG BNDF KD were apparent in both the CMS and the no-CMS control groups (Fig. $4 A, D$ ). No significant differences between groups were observed in the FST (Fig. 4C).
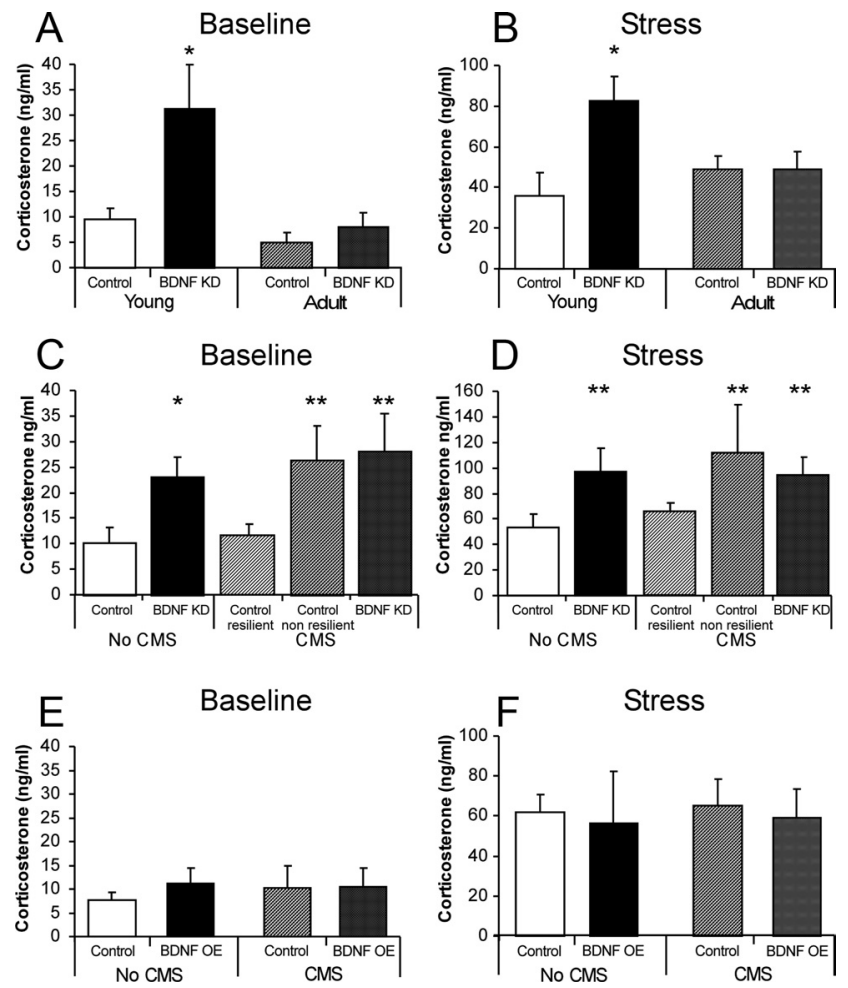

Figure 5. Corticosterone plasma levels of young and adult rats at baseline and after acute stress. Two blood samples were taken from young and adult rats that were subjected to the various BDNF and CMS manipulations. The first blood sample was taken when no stress was induced (Baseline), and the second was taken after the induction of acute stress (Stress). $\boldsymbol{A}$ and $B$ show the effect of BDNF KD on CORT levels in young and adult rats before and after the acute stress, respectively. $\boldsymbol{C}$ and $\boldsymbol{D}$ show the effect of CMS and BDNF KD on CORT levels in young rats. $\boldsymbol{E}$ and $\boldsymbol{F}$ show the effect of CMS and BDNF OE on CORT levels in adult rats. Values are mean \pm SEM $\left({ }^{*} p<0.05,{ }^{* *} p<0.01\right)$.

Experiment 3: effects of CMS and manipulations of hippocampal BDNF expression on corticosterone plasma levels of young and adult rats

Experiment $3 a$

BDNF KD in young rats induced elevations of both baseline and stress-induced CORT levels (measured in adulthood), although BDNF KD in adult rats did not cause such effects (Fig. $5 A, B)$. A two-way ANOVA showed main effects of age $\left(F_{(1,32)}=\right.$ $11.72, p<0.01)$ and LV infection $\left(F_{(1,32)}=9.16, p<0.01\right)$ on baseline CORT levels as well as a significant interaction $\left(F_{(1,32)}=5.38, p<0.05\right)$ (Fig. 5A). Post hoc Student's $t$ tests comparing the young or adult BDNF KD groups to the agematched control LV groups revealed that dDG BDNF KD induced a significant elevation in baseline plasma CORT levels in the young group $(p<0.05)$ but not the adult group (Fig. $5 A)$. Similarly, a main effect of LV injection $\left(F_{(1,31)}=8.08, p<\right.$ $0.01)$ and an interaction between LV and age $\left(F_{(1,31)}=5.03\right.$, $p<0.05$ ) were observed on stress-induced CORT levels (Fig. $5 B)$. Post hoc Student's $t$ tests revealed again that dDG BDNF $\mathrm{KD}$ induced a significant elevation in stress-induced CORT levels in the plasma of the young group $(p<0.05)$ but not the adult group (Fig. 5B).

\section{Experiment $3 b$}

The long-lasting effect of CMS in young rats on baseline and stress-induced CORT levels were evaluated in all groups that participated in experiment 2. BDNF KD induced elevations in CORT levels in young rats regardless of whether they were or 
were not subjected to CMS (Fig. $5 C, D$ ). Interestingly, in young rats that received the control LV, CMS induced similar longlasting increases of baseline and stress-induced CORT levels only in the group that was not behaviorally resilient to CMS, whereas no alterations in CORT were found in the behaviorally resilient group (Fig. $5 C, D$ ). One-way ANOVA revealed a significant effect of treatment on both baseline $\left(F_{(4,43)}=4.65\right.$, $p<0.01)$ and stress-induced $\left(F_{(4,37)}=4.12, p<0.01\right)$ plasma CORT levels. Fisher's post hoc analysis revealed that dDG BDNF KD in the young groups induced a significant elevation in both baseline and stress-induced CORT levels in both CMS and no-CMS groups relative to the no-CMS control LV group $(p<0.01)$ (Fig. 5C,D). In addition, CMS induced a significant elevation in baseline and stress-induced CORT levels in the nonresilient rats $(p<0.01)$, but not in the resilient rats, when compared with the no-CMS control LV group. Moreover, baseline and stress-induced CORT levels in the nonresilient group was significantly higher than that of the resilient group $(p<0.01)$ (Fig. 5C,D).

Experiment $3 c$

The long-lasting effect of BDNF OE and CMS in adult rats on baseline and stress-induced CORT levels were evaluated in all groups that participated in experiment 1. BDNF OE in the $\mathrm{dDG}$ of adult rats did not induce any long-lasting effects on baseline or stress-induced CORT levels. In addition, CMS in adult rats did not induce long-lasting alterations in baseline or stress-induced CORT levels (Fig. 5E, F).

\section{Discussion}

This study provides direct evidence for the role of dDG BDNF expression in resilience to the behavioral effects of chronic stress. Moreover, whereas previous studies highlighted the effect of stress or CORT on hippocampal BDNF expression, the present study, exploring the opposite interaction, shows that alterations in BDNF expression does not induce long-lasting effects on CORT levels in adult rats but that reduction in BDNF expression in young ages induces a long-lasting elevation in both spontaneous and stress-induced CORT levels.

\section{Resilience as a function of stress severity and dDG BDNF expression}

Long, but not short, maternal separations was shown previously to reduce hippocampal BDNF expression and to induce depressive-like behavior in adulthood (Francis et al., 2002; Lippmann et al., 2007). The CMS, a different paradigm applied on young (juvenile) rats, did not induce such effects (Toth et al., 2008). In the present study, surgeries in young rats were required to inject the LVs. Such surgeries may be considered another stressor in addition to the CMS procedure. Indeed, a comparison between the effect of CMS or surgeries (or their combination, using a $2 \times 2$ statistical design) on the behavioral measures of young rats showed that, unlike CMS alone or surgeries alone, the combination of the two caused a nonsignificant reduction in sucrose preference and a significant reduction in home-cage locomotion (Fig. $6 A, B)(n=$ $8-10$ per group). Similarly, a nonsignificant reduction in hippocampal BDNF expression was noted in the rats subjected to surgeries followed by the CMS procedure but not among rats that were exposed to surgeries or CMS alone (Fig. $6 C)(n=$ 5-7 per group). The association between hippocampal BDNF expression and resilience of young rats to CMS was further established after dividing young rats to behaviorally resilient

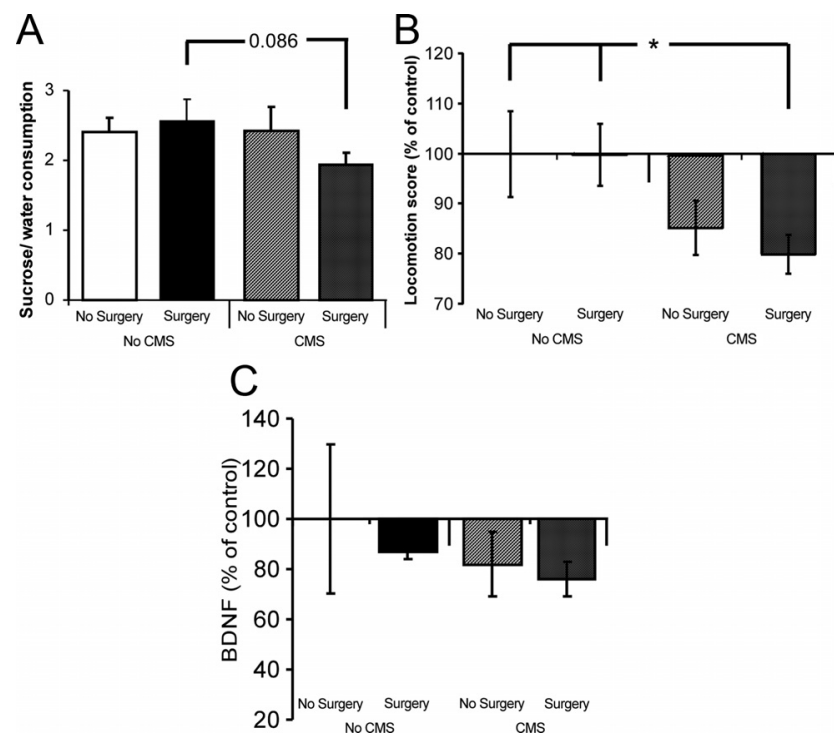

Figure 6. Effects of surgery and CMS on behavior and dDG BDNF levels in young rats. We tested whether the surgeries augmented the behavioral effects of CMS in young rats and altered dDG BDNF expression levels. Sham surgeries were or were not performed before initiation of the CMS procedure. Then, sucrose preference $(\boldsymbol{A})$ and home-cage locomotion $(\boldsymbol{B})$ were measured. In addition, the effect of CMS or surgery on dDG BDNF expression was measured (C). Values are presented as mean \pm SEM $\left({ }^{*} p<0.05\right)$.

and nonresilient, because hippocampal BDNF levels were significantly higher in the resilient young rats. In adult rats, no behavioral resilience to CMS was found (even without surgeries); sucrose preference was $3.14 \pm 0.59$ and $1.47 \pm 0.12$ for control- and CMS-treated rats, respectively $(n=7-8$ per group, $p=0.01$ ). This is consistent with previous studies showing that different forms of stress induce depressive-like behaviors (Toth et al., 2008; Gersner et al., 2010) and cause reductions in hippocampal BDNF expression (Smith et al., 1995; Toth et al., 2008; Gersner et al., 2010). Moreover, in the present study, we show that BDNF OE in the dDG of adult rats is sufficient to block the anhedonic effect of CMS and has an antidepressant effect that manifests higher FST scores. These data provide a causal interaction between BDNF expression and resilience to chronic stress. Conversely, in our control (no-CMS) groups, no correlation was found between BDNF expression and any of the behaviors (data not shown). Therefore, it seems that the relationship between hippocampal $\mathrm{BDNF}$ expression and performance in these behavioral tests shows up only after previous exposure to chronic stress.

\section{Association between allostatic overload in young animals and dDG BDNF expression}

McEwen and Wingfield (2003) have suggested previously that continued social conflict or other environmental changes or stressors drive allostasis (maintaining stability) by prolonged alterations in secretion of CORT, among other neurochemical adaptations. They define allostatic load as the cumulative cost of allostasis to the body and type 2 allostatic overload as a state in which serious pathophysiology can occur even when there is sufficient energy consumption (McEwen and Wingfield, 2003). In the case of depression triggered by childhood adversities, we suggest that reduction in $\mathrm{dDG}$ BDNF expression can be one way by which such pathological allostatic overload is mediated. Indeed, BDNF KD in the dDG of young (but not adult) rats resulted in long-lasting elevation of CORT levels. More- 
over, similar elevations in CORT levels are observed in young rats that are behaviorally nonresilient to CMS (in which BDNF levels were decreased) but not in the behaviorally resilient young rats (in which BDNF levels were not affected by CMS). We therefore suggest that genetic tendency for stress-induced reduction in $\mathrm{ADG} B \mathrm{BDNF}$ expression may underlie the individual differences observed in resilience to childhood adversities. Because BDNF is a potent regulator of plasticity and neuronal differentiation (Nestler et al., 2002; Taliaz et al., 2010), it is likely that the long-lasting effect of $\mathrm{dDG}$ BDNF reduction in young rats on CORT levels relates to the inhibitory effect of the hippocampus on the HPA axis. The circuit by which the hippocampus inhibits the HPA axis can be altered in young, still developing, rats as a result of reduced plasticity caused by the BDNF reduction.

\section{Potential mechanism for the elevation in CORT levels induced by hippocampal BDNF reduction in young animals} Chronic stress and elevated CORT levels can cause reduction in glucocorticoid receptor (GR) levels in the hippocampus, which are necessary for the negative feedback on the HPA axis (de Kloet et al., 2005; McGowan et al., 2009; Numakawa et al., 2009), thereby contributing to a pathological allostatic overload. A mechanism by which chronic exposure to CORT suppresses BDNF-induced synaptic plasticity was recently identified (Numakawa et al., 2009). In this study, chronic activation of GR inducing downregulation of the receptor suppressed BDNF-induced glutamate release, which is mediated by interaction between GR and the BDNF receptor tyrosine kinase B (Numakawa et al., 2009). An alternative mechanism for the long-lasting effect of stress on CORT levels and behavior only in nonresilient young rats may relate to regulation of GR expression. Interestingly, although not directly related to depression per se, it was reported recently that increased DNA methylation within the promoter region of exon $1_{F}$ of the $\mathrm{NR} 3 \mathrm{C} 1$, the human GR gene, was observed in suicide victims (not necessarily depressed) only if they had a history of childhood abuse (McGowan et al., 2009). This methylation results in reduced hippocampal GR levels, suggesting that the variation in the methylation status and GR expression is associated with childhood adversity (McGowan et al., 2009). It is possible, however, that the alteration in CORT levels observed in young rats could result from a more general effect on the behavioral state of the animals that was induced by the $\mathrm{dDG}$ BDNF KD. However, this possibility is not very likely because similar behavioral alterations in adult animals (induced by dDG BDNF KD) did not induce long-lasting alterations in CORT levels. Nevertheless, the potential mechanisms mediating the effect of hippocampal BDNF reduction in young rats on CORT levels will require additional investigations.

Overall, our findings that BDNF KD in the $\mathrm{dDG}$ induces depressive-like behavior in young [or adult (Taliaz et al., 2010)] animals and that BDNF OE in this subregion blocks the anhedonic effect of chronic stress emphasize the critical role of dDG BDNF in depression (or resilience to stress) and implicate hippocampal plasticity in reward-related function. These findings, when compared with the depressive-like effects of increased BDNF levels at the mesolimbic dopamine pathway and its critical role in susceptibility to stress (Krishnan et al., 2007), highlight the very different behavioral effects of BDNF alterations in different brain regions. Direct evidence is now provided that $\mathrm{dDG} B \mathrm{BDN}$ expression mediates age-linked re- silience to long-lasting behavioral and biological effects of chronic stress.

\section{References}

Altholtz LY, Fowler KA, Badura LL, Kovacs MS (2006) Comparison of the stress response in rats to repeated isoflurane or $\mathrm{CO} 2: \mathrm{O} 2$ anesthesia used for restraint during serial blood collection via the jugular vein. J Am Assoc Lab Anim Sci 45:17-22.

Charney DS (2004) Psychobiological mechanisms of resilience and vulnerability: implications for successful adaptation to extreme stress. Am J Psychiatry 161:195-216.

de Kloet ER, Joëls M, Holsboer F (2005) Stress and the brain: from adaptation to disease. Nat Rev Neurosci 6:463-475.

de Kloet ER, Derijk RH, Meijer OC (2007) Therapy insight: is there an imbalanced response of mineralocorticoid and glucocorticoid receptors in depression? Nat Clin Pract Endocrinol Metab 3:168-179.

Dhabhar FS, McEwen BS, Spencer RL (1993) Stress response, adrenal steroid receptor levels and corticosteroid-binding globulin levels: a comparison between Sprague-Dawley, Fischer 344 and Lewis rats. Brain Res 616:89-98.

Feder A, Nestler EJ, Charney DS (2009) Psychobiology and molecular genetics of resilience. Nat Rev Neurosci 10:446-457.

Fomby LM, Wheat TM, Hatter DE, Tuttle RL, Black CA (2004) Use of $\mathrm{CO} 2 / \mathrm{O} 2$ anesthesia in the collection of samples for serum corticosterone analysis from Fischer 344 rats. Contemp Top Lab Anim Sci 43:8-12.

Francis DD, Diorio J, Plotsky PM, Meaney MJ (2002) Environmental enrichment reverses the effects of maternal separation on stress reactivity. J Neurosci 22:7840-7843.

Gersner R, Dar DE, Shabat-Simon M, Zangen A (2005) Behavioral analysis during the forced swimming test using a joystick device. J Neurosci Methods 143:117-121.

Gersner R, Gordon-Kiwkowitz M, Zangen A (2009) Automated behavioral analysis of limbs' activity in the forced swim test. J Neurosci Methods 180:82-86.

Gersner R, Toth E, Isserles M, Zangen A (2010) Site-specific antidepressant effects of repeated subconvulsive electrical stimulation: potential role of brain-derived neurotrophic factor. Biol Psychiatry 67:125-132.

Grønli J, Bramham C, Murison R, Kanhema T, Fiske E, Bjorvatn B, Ursin R, Portas CM (2006) Chronic mild stress inhibits BDNF protein expression and CREB activation in the dentate gyrus but not in the hippocampus proper. Pharmacol Biochem Behav 85:842-849.

Heim C, Newport DJ, Mletzko T, Miller AH, Nemeroff CB (2008) The link between childhood trauma and depression: insights from HPA axis studies in humans. Psychoneuroendocrinology 33:693-710.

Kendler KS, Karkowski LM, Prescott CA (1999) Causal relationship between stressful life events and the onset of major depression. Am J Psychiatry 156:837-841.

Krishnan V, Han MH, Graham DL, Berton O, Renthal W, Russo SJ, Laplant Q, Graham A, Lutter M, Lagace DC, Ghose S, Reister R, Tannous P, Green TA, Neve RL, Chakravarty S, Kumar A, Eisch AJ, Self DW, Lee FS, et al. (2007) Molecular adaptations underlying susceptibility and resistance to social defeat in brain reward regions. Cell 131:391-404.

Lippmann M, Bress A, Nemeroff CB, Plotsky PM, Monteggia LM (2007) Long-term behavioural and molecular alterations associated with maternal separation in rats. Eur J Neurosci 25:3091-3098.

McEwen BS, Wingfield JC (2003) The concept of allostasis in biology and biomedicine. Horm Behav 43:2-15.

McGowan PO, Sasaki A, D’Alessio AC, Dymov S, Labonté B, Szyf M, Turecki G, Meaney MJ (2009) Epigenetic regulation of the glucocorticoid receptor in human brain associates with childhood abuse. Nat Neurosci 12:342-348.

Muscat R, Willner P (1992) Suppression of sucrose drinking by chronic mild unpredictable stress: a methodological analysis. Neurosci Biobehav Rev 16:507-517.

Nestler EJ, Carlezon WA Jr (2006) The mesolimbic dopamine reward circuit in depression. Biol Psychiatry 59:1151-1159.

Nestler EJ, Barrot M, DiLeone RJ, Eisch AJ, Gold SJ, Monteggia LM (2002) Neurobiology of depression. Neuron 34:13-25.

Numakawa T, Kumamaru E, Adachi N, Yagasaki Y, Izumi A, Kunugi H (2009) Glucocorticoid receptor interaction with TrkB promotes 
BDNF-triggered PLC-gamma signaling for glutamate release via a glutamate transporter. Proc Natl Acad Sci U S A 106:647-652.

Ridder S, Chourbaji S, Hellweg R, Urani A, Zacher C, Schmid W, Zink M, Hörtnagl H, Flor H, Henn FA, Schütz G, Gass P (2005) Mice with genetically altered glucocorticoid receptor expression show altered sensitivity for stress-induced depressive reactions. J Neurosci 25:6243-6250.

Sapolsky RM, Krey LC, McEwen BS (1984) Glucocorticoid-sensitive hippocampal neurons are involved in terminating the adrenocortical stress response. Proc Natl Acad Sci U S A 81:6174-6177.

Sapolsky RM, Zola-Morgan S, Squire LR (1991) Inhibition of glucocorticoid secretion by the hippocampal formation in the primate. J Neurosci 11:3695-3704.

Smith MA, Makino S, Kvetnansky R, Post RM (1995) Stress and gluco- corticoids affect the expression of brain-derived neurotrophic factor and neurotrophin-3 mRNAs in the hippocampus. J Neurosci 15: $1768-1777$.

Taliaz D, Stall N, Dar DE, Zangen A (2010) Knockdown of brain-derived neurotrophic factor in specific brain sites precipitates behaviors associated with depression and reduces neurogenesis. Mol Psychiatry $15: 80-92$.

Toth E, Gersner R, Wilf-Yarkoni A, Raizel H, Dar DE, Richter-Levin G, Levit O, Zangen A (2008) Age-dependent effects of chronic stress on brain plasticity and depressive behavior. J Neurochem 107:522-532.

Xu L, Holscher C, Anwyl R, Rowan MJ (1998) Glucocorticoid receptor and protein/RNA synthesis-dependent mechanisms underlie the control of synaptic plasticity by stress. Proc Natl Acad Sci U S A 95:3204-3208. 